\title{
Le caneton, réactif biologique pour le dépistage de la toxicité des tourteaux d'arachide
}

\author{
par G. THEODOSSIADES
}

En 1961, RAYNAUD (16) signaldit l'existence d'une dystrophie hépatique toxique chez les porcs à Madagascar. En 1962, le même auteur en attribuait l'étiologie aux tourteaux d'arachide. A la suite d'une mortalité importante dans certains élevages à Madagascar vers la fin 1962, nos recherches pour l'identification d'un facteur toxique dans les produits entrant dans la ration des porcs nous ont orienté vers certains lats de tourteaux d'arachide après l'expérimentation sur rat et cobaye, mais finalement la rapidité de la réponse du coneton nous a apporté la confirmation de leur rôle toxique.

En 1961, ASPLIN et CARNAGHAN (3) observent la sensibilité du caneton à un principe toxique provenant de certains Aspergillus flavus Link ex-Fries, et présent dans quelques tourteaux d'arachide.

SARGEANT, O'KELLY, CARNAGHAN et ALLCROFT (20) utilisent le caneton comme réactif biologıque en luı faisant ingérer la substance toxique isolée à partir des tourteaux à éprouver.

Nous avons voulu mettre à profit cette sensıbilité du caneton ef trouver un test simple, susceptible de rendre service, comme il l'a été dans notre cas à Madagascar, afin de pouvoir non seulement dépister les tourteaux toxiques mais aussi juger du degré de leur toxicité. En effet, écarter de l'alimentation animale tout tourteau possédant une cértaine toxicité vis-à-vis du caneton serait un luxe que l'éleveur malgache ne peut s'offrir. Pratiquement un seul tourteau, celui d'arachide, est disponible en quantité commercıale sur le marché de la Grande Ile.

II s'avère donc intéressant de tester d'une part, le degré de toxicité des tourteaux sur le coneton, et d'autre part, d'étudier la corrélatıon

Rev. Elev. Med, vet, Pays trop.; 1963, 16, n 2.

Reçu pour publicałıon : juin 1963. entre la sensibilité du caneton et celle des autres espèces.

\section{ESSAI DES TOURTEAUX D'ARACHIDE SUR LE CANETON PAR INGESTION DU PRODUIT BRUT AU MOYEN D'UN SONDAGE ESOPHAGIEN.}

\section{Matériel}

1. Une seringue de $50 \mathrm{ml}$ de capacité. Son diamètre interne doit être assez large pour faciliter d'une part, le mélange à l'intérieur avec un agitateur en verre ou une sonde canelée, d'autre part, l'expulsion de l'air afin que la seringue ne soit remplie que de la pâte constituée. Le diamètre de la seringue que nous utilisons est de $35 \mathrm{~mm}$. L'orifice de l'embout doit être élargi pour faciliter l'injection du produit. Un diamètre de $3 \mathrm{~mm}$ nous a donné satisfaction.

20 Une sonde en cooutchouc:

longueur : $160 \mathrm{~mm}$
diam. externe: $4 \mathrm{~mm}$
diam. interne : $2,5 \mathrm{~mm}$.

l'une des extrémités sera fixée sur l'embout de la seringue, ef l'autre sera terminée en biseau mousse.

3o Un broyeur (un moulin à café électrique convient).

40 Une balance.

$5^{\circ}$ Des canetons âgés de $2-3$ jours.

$6^{\circ}$ Une petıte éleveuse.

\section{Technique opératoire}

1. Marquer les canetons (par une encoche dans la membrane palmaire interdıgıtée) et les diviser en autant de lots qu'il y a d'échantillons de tourteaux à tester plus un lot témoin (nous utilisons cinq à dix canetons par lot).

1 20. Peser le tourteau finement broyé : $2 \mathrm{~g}$ par 'animal et par ingestion. 
Tableau $n^{\circ} I$

Résultats obtenus chez le caneton par ingestion forcée de tourteaux d'arachido

\begin{tabular}{|c|c|c|c|c|c|c|c|c|c|c|c|c|c|c|c|c|c|c|c|c|c|c|c|c|c|c|}
\hline & \multicolumn{2}{|c|}{ Témoin } & \multicolumn{3}{|c|}{ Tourteau $n^{0}$} & \multicolumn{3}{|c|}{ Tourteau no 2} & \multicolumn{3}{|c|}{$\begin{array}{c}\text { Tourteau no } 2 \\
\text { délijidé }\end{array}$} & \multicolumn{3}{|c|}{ Tourteau $n^{\circ} 3$} & \multicolumn{3}{|c|}{$\begin{array}{l}\text { Tourteau n० } 3 \\
\text { délipidé }\end{array}$} & \multicolumn{3}{|c|}{ Tourteau $n^{\circ} 4$} & \multicolumn{3}{|c|}{ Tourteau $n^{\circ} 5$} & \multicolumn{3}{|c|}{$\begin{array}{ll}\text { Viande } & 70 \% \\
\text { Mars } & 30 \%\end{array}$} \\
\hline $\begin{array}{l}\text { Essai } n^{\circ} 1 \\
\text { ler jour } \\
\text { 2̇me four }\end{array}$ & $\begin{array}{l}\text { A } \\
5 \\
5\end{array}$ & $\overline{8}$ & A & $\bar{B}$ & $\mathrm{C}$ & $\begin{array}{l}A \\
5 \\
0\end{array}$ & $\begin{array}{l}B \\
5\end{array}$ & $\begin{array}{l}c \\
6\end{array}$ & $\hat{A}$ & $B$ & $c$ & A & B & C & A & $B$ & $\mathrm{c}$ & A & $B$ & $\mathrm{C}$ & $A$ & $\bar{B}$ & $\mathrm{C}$ & A & $\bar{B}$ & C \\
\hline $\begin{array}{l}\text { Essai } n^{0} 2 \\
\text { ler jour } \\
\text { 2ème jour } \\
\text { 3ène jour }\end{array}$ & $\begin{array}{l}5 \\
5 \\
5\end{array}$ & & $\begin{array}{l}5 \\
4 \\
4\end{array}$ & 1 & $\begin{array}{l}5 \\
5 \\
5\end{array}$ & $\begin{array}{l}5 \\
1 \\
0\end{array}$ & $\begin{array}{l}4 \\
1\end{array}$ & $\begin{array}{l}5 \\
5\end{array}$ & & & & & & & & & & & & & & & & & & \\
\hline $\begin{array}{l}\text { Essai } n^{\circ} 3 \\
\text { 1er jour } \\
\text { 2ème jour }\end{array}$ & $\begin{array}{l}5 \\
5\end{array}$ & & $\begin{array}{l}* \\
4 \\
4\end{array}$ & & $\begin{array}{l}4 \\
4\end{array}$ & $\begin{array}{l}5 \\
0\end{array}$ & 5 & 4 & & & & & & & & & & & & & & & & & & \\
\hline $\begin{array}{l}\text { Essal no } 4 \\
\text { 1er jour } \\
\text { 2ème jour } \\
\text { 3ème jour }\end{array}$ & $\begin{array}{l}5 \\
5 \\
5\end{array}$ & & $\begin{array}{r}* * \\
7 \\
7 \\
7\end{array}$ & & $\begin{array}{l}4 \\
4 \\
4\end{array}$ & $\begin{array}{l}5 \\
4 \\
0\end{array}$ & $\begin{array}{l}1 \\
4\end{array}$ & $\begin{array}{l}4 \\
4\end{array}$ & & & & $\begin{array}{l}5 \\
5 \\
5\end{array}$ & & $\begin{array}{l}4 \\
4 \\
4\end{array}$ & $\begin{array}{l}5 \\
5 \\
5\end{array}$ & & $\begin{array}{l}4 \\
4 \\
4\end{array}$ & $\begin{array}{l}5 \\
5 \\
5\end{array}$ & & $\begin{array}{l}4 \\
4 \\
4\end{array}$ & $\begin{array}{l}5 \\
4 \\
4\end{array}$ & 1 & $\begin{array}{l}4 \\
4 \\
4\end{array}$ & $\left\{\begin{array}{l}5 \\
5 \\
4\end{array}\right.$ & 1 & $\begin{array}{l}4 \\
4 \\
4\end{array}$ \\
\hline $\begin{array}{l}\text { Essal no } 5 \\
\text { ler jour } \\
\text { 2ème jour } \\
\text { 3ème jour }\end{array}$ & $\begin{array}{l}19 \\
19 \\
18\end{array}$ & 1 & $\begin{array}{l}10 \\
10 \\
10\end{array}$ & & $\begin{array}{l}4 \\
4 \\
4\end{array}$ & $\begin{array}{l}10 \\
1 \\
0\end{array}$ & $\begin{array}{l}9 \\
1\end{array}$ & $\begin{array}{l}4 \\
4\end{array}$ & $\begin{array}{r}10 \\
2 \\
2\end{array}$ & 8 & $\begin{array}{l}4 \\
4 \\
4\end{array}$ & $\begin{array}{l}10 \\
10 \\
10\end{array}$ & & $\begin{array}{l}4 \\
4 \\
4\end{array}$ & & & & & & & & & & & & \\
\hline
\end{tabular}

* il g'agit de 4 canetons de l'essai précédent.

* Les 4 cometons de l'essad précédent plus 3 nouveaux
Colonne A nombre alanimaux en expérimentation

Colonne $B$ : nombre d'animaux morts en cours d'expérimentation

Colonne C : quantité en gxammes de tourteau ingérée par jour et par anjual (en 2 ingestions) 


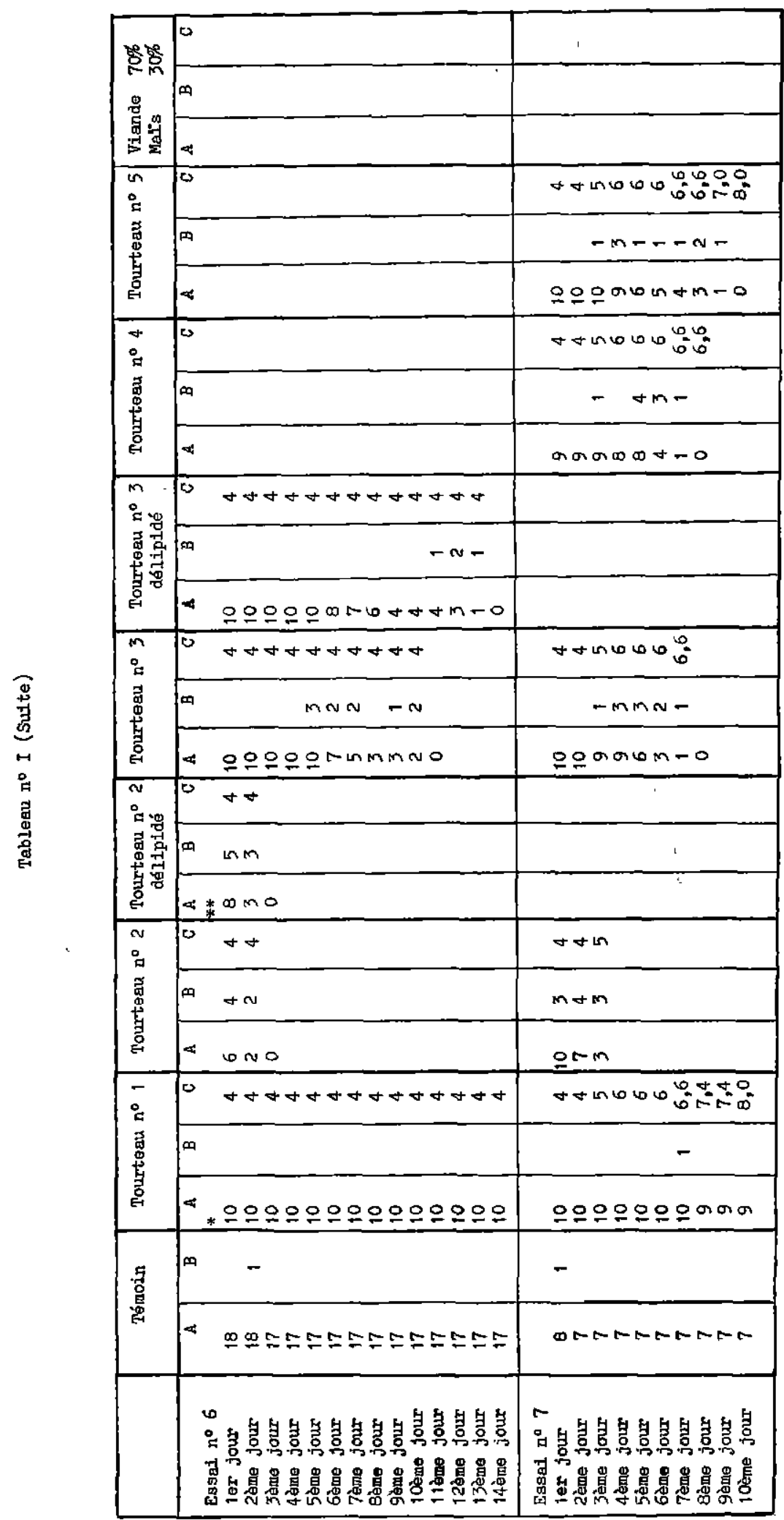

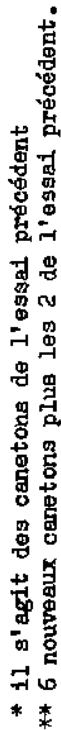


3o Mélanger à l'intérieur de la seringue le fourteau avec environ trois fois son poids d'eau pour obtenir une pâte de consistance assez fluide. Exprimer l'air. Répartir le contenu de la seringue aux canetons en le faisant ingérer par sondage œsophagien.

Le sondage ne présente pas de difficulté mais il convient, à l'entrée de la poitrine, que la main de l'opérateur oriente l'extrémité de la sonde avec doigté pour éviter blessures ou déchirures. Enfoncer ainsi la sonde au maximum (10 à $15 \mathrm{~cm}$ selon l'âge et la taille du caneton) et injecter lentement tout en retirant la sonde.

4o Mettre tous les canetons sous la même éleveuse et distribuer un aliment témoin sans tourteau.

5o Procéder à deux gavages par jour, trois heures après avoir supprımé l'aliment témoin.

\section{Résultats obtenus}

Les essais ont porté sur cing tourteaux différents. Le tableau 1 donne les résultats de sept essais réalisés. II s'en dégage que :

10 Le tourteau no 1 ne possède aucune toxicité.

$2^{\circ}$ Le tourteau no 2 possède une toxicité aiguë tuant le caneton en 24 heures à la dose de $4 \mathrm{~g}$ par jour. Remarquons que la toxicité persiste même après délipidation à l'éther.

$3^{0}$ Les tourtecux nos 3,4 et 5 possèdent une toxicité sub-ciguë. La toxicité du na 3 a persisté après délipidation à l'alcool éthylique à 960.

L'essai $n^{\circ} 7$ nous a montré que nous pouvons raccourcir la durée du test en augmentant progressivement les doses ingérées.

\section{INTERPRÉTATION DES RÉSULTATS}

\section{Origine de la toxicité}

Le facteur toxique en cause doit être le produit du métabolisme d'Aspergillus flovus, Link, isolé par SARGEANT et collaborateurs. En effet, en falsant ingérer aux canetons un extrait de tourteau obtenu par la technique décrite par. ces auteurs, nous arrivons aux mêmes résultats pour le tourteau $n^{\circ} 2$ : mortalité des canetons en moins de 24 heures. Pour les tourteaux possédant une toxicité sub-aiguè, il noùs a fallu deux ingestions, deux jours de sulte, pour obtenır 75 p. 100 de mortalité. D'autre part, le service des diagnostics du laboratoire de l'élevage, a isolé un Aspergillus flovus des tourteaux incriminés ef des arachides non traités. Tout porte done à croire que nous sommes en présence du même facteur toxique.

D'après notre enquête, la contamination par Aspergillus flavus, et surtout le degré de contamination (donc le degré de toxicité des tourteaux) proviendraient de trols causes principales :

a) Les intempéries entraînant une dessiccation lente et imparfaite des arachides mises en tas lors de la récolte, la prolıfération des champignons se trouve favorisée en climat chaud.

b) La plupart des arachides cultivées étant de variété dite « de bouche » les gousses sont ensuite triées chez les collecteurs. Les « écarts » du triage sont destinés à l'huilerie, les gousses de qualıté étant destinées à l'exportation. Le résultat en est la concentration vers l'huilerie, des «écarts» renfermant une quantité importante de gausses maisies.

c) Certains collecteurs procèdent au lavage de toutes les arachides avant leurtriage, les « écarts» non desséchés sont ensuite stockés en tas, ce qui favorise à nouveau la prolifération plus importante des champignons.

Les opérations ci-dessus expliqueraient la diversité des qualités de tourteaux produits :

o) Les arachides bien desséchées sur le champ et non triées, envoyées à l'huilerie, donneraient le tourteau non toxique : le tourtecu $n^{a} 1$ de nos essais a été obtenu de cette façon.

b) Les tourteaux $n^{\circ} 3,4$ et 5 ont été obtenus à partir d'《 écarts » de triage.

c) Le tourteau no 2 provient d'《écarts 》 mal desséchés issus d'arachides lavées avant triage, présentant une forte contamination par Aspergillus flarus.

Pour nous, le problème de la toxicité des tourteaux d'arachides se confond avec celui d'une dessiccation imparfaite des arachides après récolte et des «écarts» après lavage et stockage incorrects.

Corrélation entre le résultał des essais, qualité des tourteaux ainsi classés et leurs effets dans les rations de différentes espèces.

Le falt de déterminer la toxicité ou même le degré de toxicité d'un prodult par une épreuve 


\section{Tabloan $n^{0} \mathrm{II}$}

Résultats obtenus par incorporation de tourteaux d'arachide dans la ration de canetons

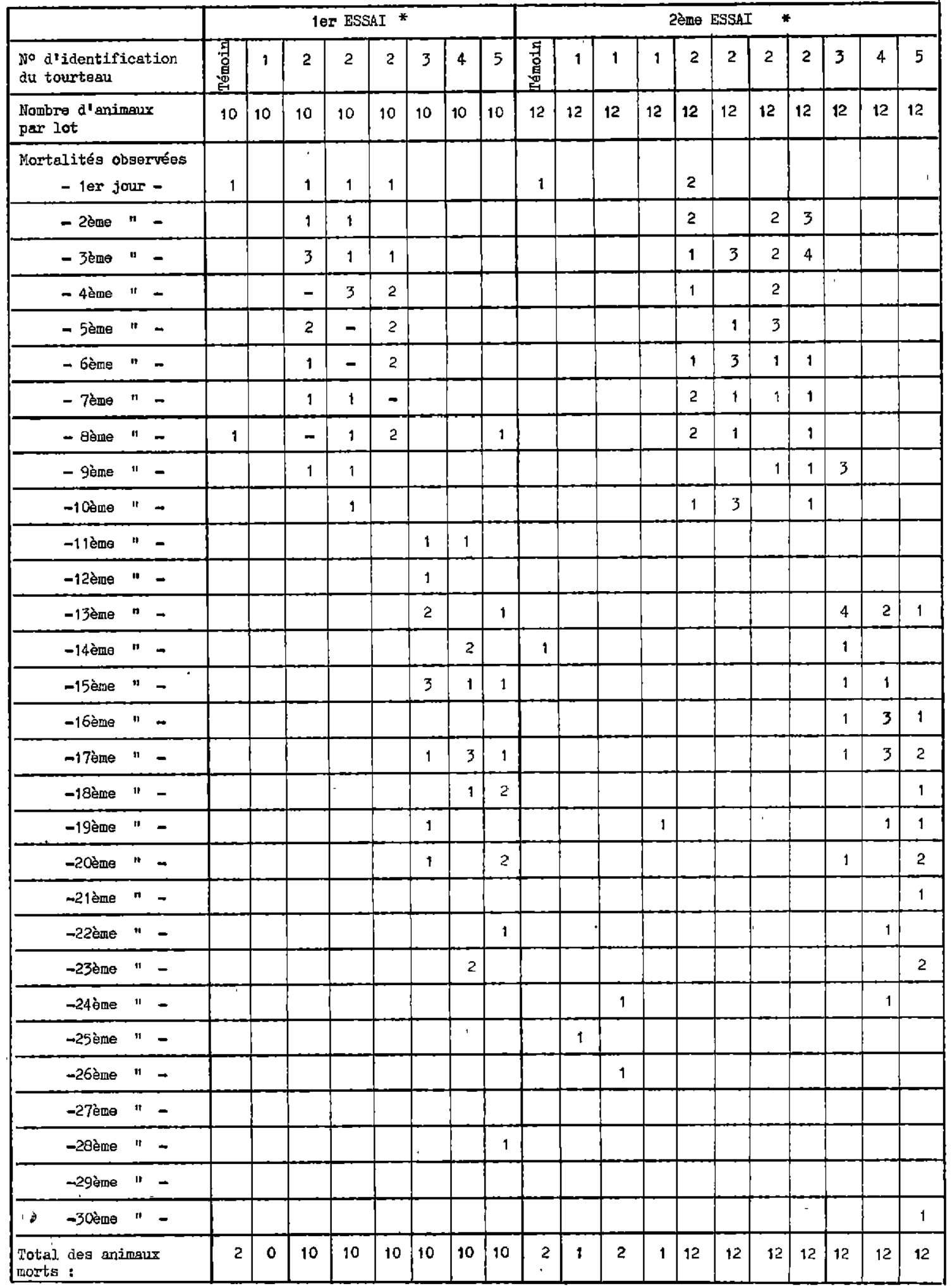

* Le taux d'incorporation des tourteaux dans la ration est uniformément de 14 p.100 
ne résoud pas les problèmes posés par son emploi en alimentation animale.

Le tableau 1 nous prouve la fidélité du test dans les conditıons expérimentales données. En est-il de même dans les conditions d'alımentation pratique et surtout, peut-on généraliser ces résultats aux autres espèces animales dans leurs conditions habituelles de production ? Possèdent-elles la même sensibilité que le caneton vis-à-vis du facteur toxique?

Il se dégage des différents travaux publiés que presque toutes les espèces $y$ sont sensibles, aınsi la toxicité des tourteaux d'arachide a été observée chez les porcs, poussins, dindons, veaux, cobayes et rats. Mais quel est le degré de cette sensibilité ? N'y a-t-il pas un taux optimum à utiliser dans les rations pour chaque qualité de tourteau ainsi classé, vis-c̄-vis de chaque espèce animale à des âges différents, pour une production zootechnique donnée? Ce taux d' « utilisation économique » permettrait de contre-balancer les effets nocifs du facteur toxique par les effets bénéfiques des éléments apportés par le tourteau, l'opération restant de ce fait rentable, à condition de lıvrer au consommateur, après vérification, un produit sain. Des recherches approfondies doivent être entreprises dans ces différentes directions pour fixer les limites d'utilisation de tels tourteaux.

Nous poursuivons des recherches dans ce sens. Des tourteaux testés sont incorporés dans des rations pour porcs en cours d'expérimentation. Dès que les résultats seront connus, ils seront communiqués. Toutefois, d'après nos observations, dans les élevages visités, c'est le tourtecu toxique no 2 qui a provoqué un taux de mortalité élevé. Des tourteaux de qualité comparable aux tourtecux $\mathrm{n}^{0 \mathrm{~s}} 3,4$ et 5 sont couramment utilisés dans les rations pour porcs, volailles et bovins, sans qu'il s'en suive de symptômes clıniques aigus. Mais il nous est difficile de nous prononcer sur la rentabilité économique d'une telle opération chez les animaux ainsı nourris qui présentent souvent, après abattage, des lésions hépatiques chroniques dont l'étiologie ne peut être à coup sûr attribuée à l'ingestion de tourteaux de cette qualité.

Chez le caneton, au contraire, la comparaison des tableaux I et ll démontre d'une part, la fidélité du test et d'autre part, que l'incorporation au taux de 14 p. 100 dans les rations du tourteau toxique $n^{\circ} 2$ et des sub-toxiques $n^{08} 3,4$ et 5 est mortelle pour cette espèce, à cet âge, Cependant, l'examen analytique de ces tableaux mef l'accent sur la nocivité à retardement des tourteaux sub-toxiques nos 3,4 et 5 , qui se manifeste après un certain temps de latence.

\section{CONCLUSION}

L'administration par sondage cesophagien au caneton constitue une épreuve fidèle permettant le dépistage des tourtecux d'arachide toxiques et leur classification à cet égard. De ces essais préliminaires, nous pouvons conclure que, tout tourteau possédant une certaine toxicité, pour le caneton, aigue ou sub-aiguë, doit être exclu de l'alimentation de cette espèce.

Par contre, pour les porcs, nos observations dans différents élevages montrent que seuls les tourteaux possédant, d'après ce test, une toxicité aiguë provoquent des signes pathologiques cliniques sub-aigüs.

Des recherches sont encore nécessarres afin d'étudier la corrélation entre les résultats de l'expérimentation chez le caneton et les effets des produits ainsı testés sur les autres animaux.

Elles permettront de fixer la fourchette économique de l'utilisation de ces tourteaux en alimentation animale. La généralisation de cette épreuve simple et rapide pourrait être alors envisagée avant leur emploi commercial.

Laboratoire Central de l'Elevage (Service de nutrition). Tonanarive.

\section{SUMMARY}

\section{The duckling as a biological indicator for the detection of toxicity in groundnut meal}

Administration to a duckling by œsophageal probe is the best means of detecting and classifying the degree of toxicity in groundnut meal. These preliminary trials show that ducklings are highly sensitive to any degree of meal toxicity. 
On the otherhand, our observations with pigs in several farms show that only highly toxic meals, according to the duckling test, provoke sub-acute clinical pathological symptoms.

Further trials are necessary to study the correlation between the results obtained with ducklings ant the effect of the meal on other animals. This will make it possible to assess the economic value of groundnut meal in animal food-stuffs.

This simple and quick technique might be generally used for toxicity assay before commercial marketıng.

\section{RESUMEN}

El anedon reactivo biologico para descubrir la foxicidad de las tortas residuales de cacahuete

La administración por sondeo esofágico al anedón constituye una prueba valedera que permite descubrir la toxicidad de las tortas residuales de cacahuete y su clasificación a este respecto. De estos ensayos preliminares puede concluirse que, para el anedón, cualquier torta residual que posea cierta toxicidad, aguda o subaguda, debe ser excluída de la alimentación.

Contrariamente, para los puercos, nuestras observaciones en distintas crianzas demuestran que únicamente las tortas que poseen, después de esta prueba, una toxicidad aguda provocan signos patológicos clínicos subagudos.

Aun serà preciso proceder a investigaciones con objeto de estudiarl a correlación entre los resultados de la experımentación en el anedón y los efectos de los productos así probados en los demás animales.

Dichas investıgaciones permitirán fijar los límites económicos de la utilización de estas tortas para la alimentación animal. La generalización de esta prueba sencilla y rápida podría ser proyectada entonces antes de su empleo comercial.

\section{BIBLIOGRAPHIE}

1. ALLCROFT (R.), CARNAGHAN (R, B. A.), SARGEANT (K.) and O'KELLY (J.). - A toxic factor in Brazilian groundnut meal. Vet. Rec., 1961, 73 (17) : 428.

2. AMARAL (L. B. S.). - Torta de amendoime morte de suinos biologico, 1961, $27: 63$.

3. ASPLIN (F. D.) and CARNAGHAN (R. B. A.). - The toxicity of certain groundnut meals for poultry with special reference to their effect on ducklings and chickens. Vet. Rec., 1961, 73, (46) : 1215-18.

4. BALEY (W. S.) and GROTH (A. H.). - The relationship of hepatitis of dogs and moldy corn poisoning in swine. J. Amer. vet. Med. ass., 1959, $134: 514$.

5. BURNSIDE (J. E.), SIPPEL (W. L.), FORGACS (J.), CARLL (W. I.), ATWOOD (M. B.) and DOLL (E. R.). - A disease of swine and cattle caused by eating moldy corn. 2. Experimental production with pure cultures of molds. Amer. J. vet. Res. 1957, 18 (69) : 817-24.
6. CARNAGHAN (R, B. A.), SARGEANT (K.). - The toxicity of certain groundnut meals to poultry. Vet. Rec., 1961, 73, (29) : 726-27.

7. COMBS (G. E.) and WALLACE (H. D.). Peanut meal as a source of protein in pig starter and grower rations. J. Anim. sci., 1962, 21 (1) : 95-7.

8. GIBSON (W. W. C.). - Toxicity of groundnut meal. Vet. Rec., 1962, 74 (3) : 99.

9. GRAY (W. V.). - Groundnut toxicity. Vet. Rec., 1961, 73 (35) : 865.

10. HORNBY (R. B.), MILLER (J. C.) and DABEL (J. S.). - Toxicity of groundnut meal. Vet. Rec., 1962, 74 (1) : 52.

11. KOHLER (H.) and SWOBODA (R.). - Durch Erdnusschote ausgelốste leberzirrhosen bei Enten. Vien. Tierärztl. Monatsschr. 1962, 49 : 205.

12. LANGASTER (M. C.), JENKINS (F. P.) and PHILIP (J. M.). - Toxicity associated with certain samples of groundnuts. Noture., 1961, 192 : 1095-6. 
13. LOOSMORE (R. M.) and MARKSON (L. M.). -Poisoning of cattle by Brazilian Groundnut meal. Vet. Rec, 1961, 73 (33) : 813-4.

14. LOOSMORE (R. M.) and HARDING (J. D. J.). - A toxic factor in Brazilian groundnut causing liver damage in pigs. Vet, Rec., 1961, 73, (49) : 1362-4.

15. PATERSON (J. S.), CROOK (J. C.), SHAND (A.), LEWIS (G.) and ALLCROFT (R.).Groundnut toxicity as the cause of exudative hepatitis (Oedema disease) of guinea-pigs. Vet. Rec., 1962, 74 (22) : 639.

16. RAYNAUD (J. P.). - Une épidémie d'hépatite-cirrhose du porc à Madagascar. I. Efude des tests hépatiques chez le porc et utilisation de la vitesse de sédimentation pour un diagnostic précoce. Rev. Elev. Med. vet. Pays, trop., 1961, 14 (4) : 429-37.

17. RAYNAUD (J. P.). - Une dystrophie hépatique toxique du porc à Madagascar. Il. Etude clinique, lésıons, reproduction expérimen- tale par ingestion de tourteau d'arachide. Rev. Elev. Med, vet. Pays, trop., 1963, 16 (1). 18. RICHMOND (J. W.), SUTCHIFFE (N. H.), DANIELS (N. W. R.), EGGIT (P. W. R.) and COPPOCK (J. B.). - Factors other than groundnut relating to « turkey $X$ disease ». Vet. Rec, 1962, 74 (18) : 544.

19. SARGEANT (K.), ALLCROFT (R.) and CARNAGHAN (R. B. A.). - Groundnut toxicity.

20. SARGEANT (K.), O'KELLY (J.), CARNAGHAN (R. B. A.) and ALLCROFT (R.).-The assay of a toxic principle in certain groundnut meals. Vet. Rec, 1961, 73 (46) : 1219-22.

21. SARGEANT (K.), SHERIDAN (A.), O'KELLY (J.) and CARNAGHAN (R. B. A.). - Toxicity associated with certains samples of groundnuts. Noture, 1961, $192: 1096$.

22. ZWART (D.). - Liver cirrhosis in pigs in Ghana. Thèse Utrecht 1962. 